\title{
Alter
}

Revue de phénoménologie

$21 \mid 2013$

La Vie

\section{La vie d'un soi, vie dans un monde}

Michel Henry et le jeune Heidegger

\section{Claudia Serban}

\section{OpenEdition}

\section{Journals}

Édition électronique

URL : http://journals.openedition.org/alter/847

DOI : 10.4000/alter.847

ISSN : 2558-7927

Éditeur :

Association ALTER, Archives Husserl (CNRS-UMR 8547)

\section{Édition imprimée}

Date de publication : 1 novembre 2013

Pagination : 187-203

ISBN : 978-2-95-223749-9

ISSN : 1249-8947

Référence électronique

Claudia Serban, «La vie d'un soi, vie dans un monde », Alter [En ligne], 21 | 2013, mis en ligne le 01 juin 2019, consulté le 07 juillet 2019. URL : http://journals.openedition.org/alter/847 ; DOI : 10.4000/ alter.847 


\section{LA VIE D'UN SOI, VIE DANS UN MONDE. MICHEL HENRY ET LE JEUNE HEIDEGGER}

Claudia Serban

Pour une approche phénoménologique de la vie, ce qui est en question, ce n'est pas le quid, mais le comment: comment la vie s'éprouve elle-même. Le phénomène de la vie est donc de prime abord son expérience, car c'est là que la vie non seulement apparaît, mais s'apparaît à elle-même. Le préalable méthodologique d'une telle enquête est le va et vient constitutif entre la première et la troisième personne, entre le vivant que je suis et qui éprouve la vie en soimême, et cette vie dont je fais l'expérience mais dont je ne détiens pas le monopole, qui est chose commune et qui renvoie donc aussi aux expériences des autres.

Pour explorer la manière dont la vie s'apparaît et s'éprouve ellemême, nous nous appuierons ici sur deux aperçus qui nous semblent exemplaires chacun à sa façon : celui qu'en donne le jeune Heidegger (nous visons ainsi son premier enseignement fribourgeois, allant de 1919 à 1923) et celui que l'on trouve chez Michel Henry. Le fil conducteur de notre lecture, qui nous permettra aussi d'évaluer les positions respectives de ces deux auteurs, sera la distension de l'expérience de la vie entre l'ipséité et la mondanéité. Le rapprochement ${ }^{1}$

\footnotetext{
${ }^{1}$ Les bases d'un tel dialogue ont déjà été posées par Jean Greisch, qui parle du « débat inévitable " entre phénoménologie matérielle et herméneutique de la vie facticielle (J. Greisch, L'Arbre de Vie et l'Arbre du savoir. Les racines phénoménologiques de l'herméneutique heideggérienne (1919-1923), Paris, Cerf, 2000, p. 34, ainsi que «L'Arbre de la Vie et l'Arbre du savoir », in Michel Henry, l'épreuve de la vie, éd. A. David et J. Greisch, Paris, Cerf, 2000, p. 383-406). Voir aussi J.-Cl. Gens, "Heidegger à Fribourg: le "frémissement" d'un savoir de la vie", in Michel Henry, l'épreuve de la vie, op. cit., p. 319-332, et Sylvain Camilleri, Phénoménologie de la religion et herméneutique théologique dans la pensée du jeune Heidegger, Dordrecht, Springer, 2008, en particulier p. 179-181 et p. 464-468. Qu'il nous soit permis de renvoyer en outre à notre contribution : «Michel Henry und der frühe Heidegger als Lebensphänomenologen » (in Sein, Existenz, Leben :
} 
implicite que nous proposons ainsi entre Henry et Heidegger a néanmoins de quoi surprendre : tout d'abord, en ce qui concerne Heidegger, parce que le fait d'en faire un phénoménologue de la vie peut passer pour un anachronisme naïf eu égard à la distance explicite que Sein und Zeit prend, non seulement à l'égard de toute Lebensphilosophie, mais aussi par rapport à l'idée d'une considération de la vie indépendamment du Dasein ${ }^{2}$. Plus loin encore, c'est Michel Henry luimême (qui non seulement ne semble pas avoir eu une connaissance directe des cours du jeune Heidegger du début des années 20, mais, surtout, a élaboré sa propre phénoménologie dans une intention expressément polémique, comme une réponse critique à l'analytique du Dasein) qui semble s'esquiver d'emblée face à toute perspective de confrontation ${ }^{3}$.

Pourtant, notre objectif n'est pas d'avancer une lecture comparatiste de deux corpus, mais de chercher à deux endroits différents des réponses à une même constellation de problèmes ${ }^{4}$. En effet, on sait que ce que thématise le jeune Heidegger est la vie facticielle - une vie que, dans le sillage de Dilthey, il conçoit comme historique et expressive, alors que Michel Henry s'intéresse à la vie comme corporelle et affective - ces deux caractères étant intimement inscrits dans la notion, centrale pour lui, d'auto-affection. Mais les questions qui surgissent à partir de ces deux façons de déterminer la vie, de la rendre accessible et descriptible, se laissent regrouper autour de deux axes qui leur sont communs. Tout d'abord, la question de l'ipséité : dans quelle mesure l'expérience que fait la vie se laisse caractériser comme

\footnotetext{
Michel Henry und Martin Heidegger, éd. S. Grätzel et F. Seyler, Fribourg/Munich, Karl Alber Verlag, 2013, p. 107-128), dont nous proposons ici un prolongement.

${ }^{2}$ Cf. Heidegger, Sein und Zeit (1927), Tübingen, Max Niemeyer Verlag, 2001, § 10, p. 50 : «La vie est un mode d'être spécifique, mais il n'est essentiellement accessible que dans le Dasein ». Nous reproduisons ici la traduction d'Emmanuel Martineau (édition numérique hors-commerce). Pour la critique constante de cette thèse d'Être et temps par Michel Henry, voir par exemple Phénoménologie de la vie, tome I : De la phénoménologie, Paris, PUF, coll. "Épiméthée», 2003 abrégé par $P V I-$, p. 131 : « Loin que nous n'ayons accès à la vie que dans le Dasein, c'est dans la vie seulement et par elle que nous avons accès à lui ».

${ }^{3}$ Comme l'écrit Jean-Claude Gens, la question se pose de savoir «si ce n'est pas une simple homonymie, parfois étonnante, entre les conceptualités des pensées henryenne et heideggérienne de la vie, qui donne l'illusion de leur proximité » (J.-Cl. Gens, « Heidegger à Fribourg : le "frémissement" d'un savoir de la vie ", art. cit., p. 320-321). Jean Greisch formule à son tour le même problème : "Comment déterminer la proximité, mais aussi l'écart, entre la conception heideggérienne et la conception henryenne de la vie? » (J. Greisch, L'Arbre de vie et l'Arbre du savoir, op. cit., p. 208).

${ }^{4}$ Nous espérons réussir ainsi à échapper au « dialogue de sourds » qu'identifie François-David Sebbah entre les "phénoménologies du Monde » et la phénoménologie de la vie (F.-D. Sebbah, "En deçà du monde? À propos de la philosophie de Michel Henry », Les études phénoménologiques, no 39-40, 2004, p. 81-94, p. 82).
} 
expérience de soi et en quoi est-elle expérience de quelque chose comme un soi ? D'autre part, c'est la question du rapport de la vie à ce qui n'est pas elle mais l'entoure qui se pose: il s'agit alors de savoir si l'expérience de la vie, a fortiori au cas où elle suppose nécessairement quelque chose comme une dimension d'ipséité, est aussi, de façon constitutive et tout aussi originairement, expérience $\mathrm{du}$ monde. Cette dernière question est tout sauf anodine, si l'on songe au reproche d'acosmisme dont les deux auteurs auxquels nous nous intéressons ici ont fait l'objet: Michel Henry, par le fait de proposer explicitement une caractérisation de la vie comme acosmique, et Heidegger, de façon plus subtile, à cause du fait d'avoir finalement pensé le monde comme une structure existentiale du Dasein ${ }^{5}$. Nous $\mathrm{n}^{\prime}$ allons pas nécessairement chercher dans l'objection d'acosmisme un terrain de rencontre entre les deux philosophes (même si cette objection nous invite tout de même, de façon plutôt paradoxale et ironique, à nuancer la lecture que Michel Henry fait constamment de la pensée de Heidegger comme " phénoménologie du monde »), car il est clair que d'autres divergences viendraient aussitôt miner le rapprochement : pensons seulement à la tentation, somme toute puissante bien qu'évidemment caricaturale, d'opposer Michel Henry et Heidegger comme représentants des philosophies de l'immanence ou de la transcendance. Dans sa propre interprétation de Heidegger, Michel Henry a d'ailleurs constamment souligné cette contrariété, jusqu'à lui faire correspondre l'opposition entre Apollon, le « dieu de la lumière », et Dionysos, qui « $n^{\prime}$ a pas de monde $»^{6}$. Fondée ou non, cette interprétation ne doit pourtant pas nous empêcher de prendre la mesure de certaines affinités tout à fait fondamentales qui réunissent les deux phénoménologues surtout dans leur rapport critique à Husserl et qui manifestent sans doute quelque chose comme une nécessité interne de la chose elle-même - cette chose qu'ils s'efforcent tous deux à penser : la vie -, tel par exemple le primat de l'affectivité au détriment de la réflexion ou de la théorie.

\footnotetext{
${ }^{5}$ Un des premiers à formuler cette objection a été son ancien étudiant Karl Löwith. Voir son «Zu Heideggers Seinsfrage: Die Natur des Menschen und die Welt der Natur » (1969), in Sämtliche Schriften, tome 8, Stuttgart, Metzler, 1984, p. 288 : "Depuis Sein und Zeit, on parle fréquemment du Dasein comme être-au-monde, mais on oublie de dire que le monde du Dasein n'est pas le cosmos ordonné » (cité par Robert Brisart dans son ouvrage La phénoménologie de Marbourg, ou la résurgence métaphysique chez Heidegger à l'époque de Sein und Zeit, Bruxelles, Facultés universitaires Saint-Louis, 1991, qui réactive à plusieurs reprises ce reproche d'acosmisme - par exemple, p. 15, 153, 163, etc.).

${ }^{6}$ Michel Henry, Phénoménologie de la vie, tome III : De l'art et du politique, Paris, PUF, coll. «Épiméthée », 2004 - abrégé par PV III -, p. 287.
} 


\section{La vie et le soi d'avant la réflexion}

Il est en effet fort significatif qu'au début de son propre opus magnum de 1963, L'essence de la manifestation, qui peut être lu d'un bout à l'autre comme une magistrale réplique à Sein und Zeit, Michel Henry s'assigne d'entrée de jeu une tâche formulée par Heidegger lui-même : celle de penser le "sens d'être de l'ego ». Certes, il n'est pas question pour Henry ce faisant de répéter $\hat{E}$ tre et temps. Sa thèse sera bien plutôt que Heidegger a manqué à son tour, comme il le reproche lui-même à Husserl et à Descartes, le sens d'être de l'ego, en élaborant, à la place d'une " ontologie de l'ipséité » ${ }^{7}$, une « ontologie du monde ». C'est là une opposition que Michel Henry a soulignée avec insistance lorsque, invité en septembre 1966 à Copenhague pour un colloque international consacré à Kierkegaard, il a pris la parole après la conférence du philosophe danois Knud Ejler Løgstrup, qui avait avancé une lecture kierkegaardienne de Heidegger. Avouant sa surprise face à ce rapprochement, Michel Henry affirme à cette occasion sa conviction qu'il n'y a " pas deux ontologies plus fondamentalement différentes que celles de Kierkegaard et de Heidegger » : une « ontologie de la subjectivité et de l'intériorité radicale », ou encore de l'ipséité d'une part, et une "ontologie de l'avènement impersonnel de l'être », "de l'objectivité transcendantale, de l'extériorité non moins radicale $»^{8}$, de l'autre. On peut aisément deviner de laquelle des deux entreprises Henry se sentait le plus proche. Ce jugement pourrait néanmoins être nuancé si l'on tient compte du rôle formateur tout à fait décisif que Kierkegaard a joué pour Heidegger. Pensons seulement à la fameuse déclaration de 1923, que l'on cite presque toujours de façon tronquée : «Compagnon dans la recherche $\mathrm{m}^{\prime}$ a été le jeune Luther et modèle Aristote, que ce dernier détestait. Kierkegaard m'a donné des impulsions et les yeux m'ont été implantés par Husserl $»^{9}$. Kierkegaard et Husserl : ces sources et références communes aux deux phénoménologies de la vie élaborées par le jeune Heidegger et Henry - sous la forme, d'une part, d'une herméneutique de la vie facticielle, et de l'autre, d'une phénoménologie

\footnotetext{
${ }^{7}$ Michel Henry, L'essence de la manifestation (1963), Paris, PUF, coll. «Épiméthée ", 19902 (abrégé par $E M)$, p. 757 : «Une définition du héros vient se substituer à la détermination ontologique de l'essence de l'ipséité ».

8 Michel Henry, «À propos de Kierkegaard et Heidegger », in Michel Henry, éd. J.-M. Brohm et J. Leclercq, Lausanne, L'Âge d'Homme, 2009, p. 70-73, p. 71.

${ }_{9}^{9}$ GA 63, p. 5 (Nous citons les cours de Heidegger selon l'édition intégrale parue aux éditions Klostermann à Francfort-sur-le-Main que nous abrégeons conformément à l'usage par le sigle GA suivi du numéro du volume).
} 
matérielle de la vie incarnée - suffiront peut-être à donner une certaine légitimité à notre entreprise.

Mais partons de points de rapprochement plus concrets, comme par exemple - et avant tout - la parenté profonde qui existe entre la percée de Heidegger dans la phénoménologie en 1919 et le « renversement de la phénoménologie » que propose Henry dans son dernier grand ouvrage phénoménologique, Incarnation, paru en 2000 : il s'agit à chaque fois, en brisant le "primat du théorétique », de ménager un accès à un domaine plus originaire que celui des vécus ou des objectivités corrélatives de ces vécus - celui d'une vie pré-réflexive et inobjectivable. Pourtant, si les deux philosophes rejettent toute approche objectivante de la vie, la prudence critique de Heidegger est sur ce point sans doute plus grande, puisqu'il récuse en même temps la saisie de la vie par le concept de subjectivité : pour lui, «l'objectivation comme la subjectivation sont des manières de théorétiser ${ }^{10}$. Alors que, selon cette perspective, il importe surtout de ne pas faire passer la phénoménologie de la vie pour une nouvelle philosophie de la subjectivité, Michel Henry essaie au contraire, par la considération phénoménologique de la vie, de faire sortir le traitement philosophique de la subjectivité de sa crise ${ }^{11}$. En faisant sien le vocabulaire de la subjectivité, Henry accepte aussi celui de l'intériorité, tandis que Heidegger se tiendra toujours à distance d'une approche de la vie à partir de la catégorie d'immanence - comme d'ailleurs, au tournant des années 20, à partir de celle de transcendance. Il écrit par exemple : «la considération de la sphère entière de la vie pure n'est ni intérieure ni extérieure, ni transcendante ni immanente $\gg^{12}$. Par delà cette volonté de se démarquer d'une conceptualité trop traditionnelle qui est d'ailleurs encore celle de Husserl, il s'agit peut-être aussi d'un refus d'opérer cette séparation abstraite ou de choisir entre intérieur et extérieur. C'est pourquoi dans un cours ultérieur, dans un contexte qui ne permet pas tout à fait de décider dans quelle mesure il parle en son propre nom, Heidegger fera référence à la «transcendance immanente de la vie $»^{13}$.

\footnotetext{
10 « Leben nicht Objekt, ist aber auch nicht philosophisch erfasst durch Verankerung im Subjekt, durch ichliche Betrachtungsweisen. Objektivierung und Subjektivierung sind Theoretisierungsweisen. Auch immanente Reflexion geht auf objektivierte Transzendenzen » (GA 58, p. 145; cf. ibid., p. 236).

${ }^{11}$ Voir à ce sujet l'article de 1966: "Le concept d'âme a-t-il un sens ?" (Revue philosophique de Louvain, 64, 1966, p. 5-33, repris dans PVI, p. 9-38).

12 GA 58, p. 253.

${ }_{13}$ GA 59, p. 70.
} 
Tout en s'efforçant de désactiver l'opposition classique entre immanence et transcendance, Heidegger est peut-être encore en train d'œuvrer à une continuation de la tentative husserlienne de penser une «transcendance dans l'immanence ${ }^{14}$. Cela n'est évidemment pas sans rapport avec la question de savoir comment s'articulent, au sein de l'expérience de la vie, l'expérience de soi et l'expérience du monde. Car manifestement, le refus du vocabulaire de la subjectivité et de l'intériorité ne dénote ici pas un manque d'intérêt pour la thématique de l'ipséité, ni le refuge dans une pensée de l'objectivité. Bien au contraire, à cette époque où, en cherchant à assigner un sol pré-théorétique à son entreprise, il est soucieux avant tout de se situer en deçà de l'objectivation (jusqu'à prendre, sur ce point, la défense de Husserl contre Natorp), Heidegger explore le domaine d'une vie qui est toujours vie d'un soi. Se garder d'objectiver ce domaine par le regard théorique, c'est aussi refuser de reconnaître comme unique voie méthodique d'accès à la vie et au vécu, à l'instar de Natorp (et peut-être finalement de Husserl lui-même, souvent fidèle à Brentano sur ce point), la réflexion. Par là, Heidegger préfigure à coup sûr la critique de la réflexion que Michel Henry dirigera, dans Incarnation notamment, contre Husserl et Fink ${ }^{15}$. Il anticipe en outre, surtout, sur la manière de déterminer l'accès pré-théorétique et pré-réflexif à la sphère de la vie comme étant de nature affective ou "émotionnelle (emotionaler Art) $»^{16}$.

Le rôle de l'affectivité dans la pensée de Heidegger ne se démentira pas au moins jusqu'à Sein und Zeit, et Henry n'a pas pu manquer d'en prendre acte et connaissance. Un important paragraphe de L'essence de la manifestation (le § 65) traite précisément du « pouvoir de révélation de l'affectivité selon Heidegger ${ }^{17}$, même $s^{\prime}$ il ne $s^{\prime}$ agit nullement d'un éloge, mais au contraire d'une critique de la concep-

\footnotetext{
${ }^{14}$ Cf. Niall Keane «Why Henry's Critique of Heidegger Remains Problematic: Appearing and Speaking in Heidegger and Henry ", Studia phaenomenologica, vol. IX, 2009, p. 193-212, p. 211.

15 Voir par exemple Michel Henry, Incarnation, Paris, Seuil, 2000 - abrégé par $I$-, p. 104, 105, 121, 132. À ce sujet, qu'il nous soit permis de renvoyer à notre étude : "Conscience impressionnelle et conscience réflexive : Husserl, Fink et les critiques phénoménologiques ", Revue philosophique de la France et de l'étranger, no 4/2012, p. 473-493.

${ }_{16}$ GA 58, p. 39. De façon suggestive, Henry cite à l'avant-dernière page de L'essence de la manifestation deux vers de Hölderlin qui pourraient tout aussi bien servir de motto à l'entreprise de Heidegger: "Peu de savoir mais beaucoup de joie/ Tel est le lot des mortels" (EM, p. 861). Comme le remarque Jean Greisch, «Heidegger partage la méfiance à l'égard de la représentation qui joue un rôle fondamental dans la phénoménologie matérielle de Michel Henry » (J. Greisch, L'Arbre de vie et l'Arbre du savoir, op. cit., p. 285).

${ }^{17} \mathrm{La}$ traduction anglaise (par Girard J. Etzkorn) de ce paragraphe représente d'ailleurs la contribution de Michel Henry au volume collectif Martin Heidegger. Critical Assessments, tome I : Philosophy, éd. Ch. Macann, Londres, Routledge, 1992, p. 354-369.
} 
tion heideggérienne de la Befindlichkeit comme intimement liée à la temporalité et à la transcendance ${ }^{18}$. Cependant, dans ses cours du début des années 20 où la transcendance n'est pas encore un Grundbegriff de sa pensée, Heidegger se trouve engagé bien plutôt dans une entreprise de dépassement et de refondation du rapport intentionnel à soi qui n'est pas sans affinités avec la démarche henryenne $^{19}$. Comme en témoigne par exemple le premier cours qu'il prononce en arrivant à Marbourg, pendant le semestre d'hiver 19231924, à propos de la Verdrehung (que l'on rend sans doute le mieux par détournement, mais qui renvoie aussi à l'idée de torsion et de déformation) :

Ce phénomène de la Verdrehung, un phénomène fondamental qui a longtemps été identifié à la réflexion, a pour nous le caractère d'un fil conducteur méthodique, dans la mesure où à partir de ce phénomène le caractère fondamental de la conscience, l'intentionnalité, est conduit et renvoyé à ses limites, aux limites de sa fonction d'explicitation. En même temps, ce phénomène est un sol structurel sur la base duquel des phénomènes comme la joie, la peur, la tristesse, l'angoisse deviennent susceptibles d'être explicités - des phénomènes qu'on manque lorsqu'ils sont déterminés comme intentionnalité. Je ne peux pas saisir le phénomène de l'angoisse comme fait d'être en rapport avec quelque chose, mais il s'agit d'un phénomène du Dasein lui-même $e^{20}$.

On voit s'esquisser ici un accès à soi - ou au Dasein propre, pourrait-on dire, pour respecter la terminologie de ce cours où s'amorce déjà la transition de l'herméneutique de la vie facticielle à l'analytique du Dasein - affectif et pré-intentionnel. Si, sur ce point, Michel Henry est susceptible de trouver dans le jeune Heidegger un ancêtre inattendu et insoupçonné, on sait d'autre part que la fondation henryenne de l'intentionnalité aboutit à la position d'une «dualité de l'apparaître » qui instaure une hétérogénéité sans reste entre l'apparaître à soi affectif de la vie ${ }^{21}$ et $l^{\prime}$ apparaître sensible du monde. Dans la perspective de cette dualité, il n'est assurément pas facile, comme nous le verrons, de tenir ensemble la vie comme expérience de soi et la vie comme expérience du monde. Si Heidegger est peut-être moins exposé à cette difficulté, c'est sans doute parce que pour lui, les

\footnotetext{
${ }^{18}$ Cf. EM, p. 743 : « Le pouvoir de l'affectivité [...] est le pouvoir de la transcendance ».

${ }^{19}$ Voir à ce sujet Michel Henry, "Phénoménologie non-intentionnelle : une tâche de la phénoménologie à venir ", in L'intentionnalité en question, éd. D. Janicaud, Paris, Vrin, 1995, p. 383-397, et notre contribution: "Michel Henry et la question du fondement de l'intentionnalité », Bulletin d'analyse phénoménologique, no 8, 2010, p. 284-304.

${ }^{20} \mathrm{GA} 17$, p. 288, nous traduisons.

${ }^{21}$ Cf. EM, p. 596 : «Toute vie est par essence affective, l'affectivité est l'essence de la vie ».
} 
phénomènes affectifs de l'existence - comme l'angoisse, la peur, la tristesse et la joie, qu'il mentionne dans le passage cité - ne renvoient pas à une sphère égoïque spécifique, mais à un soi qui, nous le verrons, a toujours sa Selbstwelt (son monde propre).

\section{Affectivité et facticité}

En essayant de penser l'affect dans sa concrétude, Henry est en revanche conduit à une accentuation de la dimension d'immanence de l'ipséité, et ce au moyen d'une réactivation de la phénoménologie husserlienne de la hylé : avoir un affect, sentir, c'est se sentir, s'affecter ou s'éprouver soi-même (en cela, le concept henryen d'auto-affection rend rigoureusement compte de sa conception de l'expérience de soi). Affect et sensation ou impression sont toujours donnés ensemble. De cette co-donation, de cette intrication entre le sentir et le se sentir, Henry tire la double conséquence d'un soubassement affectif de chaque impression, de chaque perception, et d'une matière impressionnelle de chaque affect. C'est ce que suggère déjà l'expression de Descartes dans la deuxième Méditation métaphysique : "videre videor ", abondamment reprise et commentée par Henry ${ }^{22}$. (Notons en passant que l'herméneutique de la vie facticielle du jeune Heidegger reste quant à elle très éloignée de toute réviviscence du cartésianisme et se nourrit bien au contraire plutôt de sa critique constante; et cette divergence de filiation entre Husserl et Henry d'une part et Heidegger d'autre part est loin d'être sans importance.) La sensation fournit la matière de l'affect, que Michel Henry nomme aussi impression et qui désigne son contenu concret. La phénoménologie husserlienne de l'impression, qui connaît un premier sommet dans les Leçons sur la conscience intime du temps données à Göttingen en 1905, reste à cet égard une référence incontournable. Elle n'est pourtant nullement assumée telle quelle, car le traitement husserlien de l'impression originaire et son inscription dans le réseau intentionnel des rétentions et des protensions est soupçonné de produire aussitôt son altération. C'est pourquoi Henry se propose, dans l'ouvrage éponyme, de substituer à la phénoménologie hylétique de Husserl une phénoménologie matérielle, qui se propose de prendre en compte plus radicalement l'Urimpression. Pour le jeune Heidegger en revanche, qui, comme nous l'avons vu, refuse de faire sien le vocabulaire de ${ }^{22}$ Michel Henry, Généalogie de la psychanalyse, Paris, PUF, coll. «Épiméthée », 1985 - abrégé par
GP -, chapitre I. 
l'immanence (la hylé relevant chez Husserl, on le sait, de l'immanence réelle), le concept même d'un datum hylétique n'a pas de pertinence phénoménologique ${ }^{23}$ pour une description de la vie. Ainsi peut-il écrire : "Vécus - je ne pense pas par là à des data hylétiques, mais à des situations concrètes de la vie (konkrete Situationen des Lebens) ${ }^{24}$.

Un autre sens du concret, qui ne se nourrit pas de la teneur matérielle et impressionnelle du sentir, anime donc la considération heideggérienne de la vie et des vécus. Pour notre propos, cela veut aussi dire que l'expérience de soi de la vie, tout en ayant une indéniable dimension affective, ne se laissera pas entièrement reconduire à la structure que manifeste le «videre videor» cartésien, à l'intrication de l'impression et de l'affect. Quelles que soient ses motivations, le refus de s'engager sur un tel terrain est peut-être aussi symptomatique de la sous-détermination de ce que serait dans ce contexte une phénoménologie de la perception, voire de l'impression. Car il est certain que, tout en n'étant pas assimilable à une pure introspection réflexive ou à une saisie de soi intellective, l'expérience de la vie selon le jeune Heidegger n'est pas de prime abord expérience sensible, même si, par sa référence au vécu, elle reste assurément encore épreuve sentie. Mais elle a une autre sorte de concrétude, que l'on peut préciser à l'aide de ce concept clé qu'est la facticité. La facticité de la vie, dans sa spécificité, se tient à égale distance de la choséité et de la pureté d'une conscience transcendantale. Plus positivement, Heidegger en donne deux caractères qui disent à chaque fois beaucoup de ce qu'est pour lui l'expérience de la vie : l'historicité et l'expressivité. Il s'agit de deux dimensions que la phénoménologie henryenne de la vie n'a jamais entièrement fait siennes ${ }^{25}$, comme nous aurons l'occasion de le voir.

L'historicité, tout d'abord, fournit la première et sans doute la plus importante caractérisation positive de la facticité chez le jeune Heidegger ${ }^{26}$. Elle fait référence à la processualité et à la mobilité intrinsèque de toute vie, donc à l'histoire que "nous sommes nousmêmes ${ }^{27}$. Comme le disent toujours les Remarques critiques sur la Psychologie des visions du monde de Jaspers (1919-1921): «Il se

${ }^{23}$ Cf. GA 58, p. 182.

${ }^{24}$ GA 58, p. 188.

${ }^{25}$ Nous pouvons sur ce point, avec Jean Greisch, « nous demander si ce n'est pas cette ouverture à l'expressivité qui marque la différence entre la phénoménologie herméneutique heideggérienne et la phénoménologie matérielle de Michel Henry qui entend l'autosuffisance de la vie comme une auto-affection » (J. Greisch, L'Arbre de la vie et l'Arbre du savoir, op. cit., p. 60).

${ }^{26}$ Cf. GA 60, p. 9. Voir à ce sujet la contribution de Sophie-Jan Arrien, « Vie et Histoire (Heidegger 1919-1923) », Philosophie, no 1, 2001, p. 51-69.

${ }_{27}$ GA 9, p. 34. 
pourrait que les phénomènes de la vie soient selon leur sens fondamental "historiques" et accessibles seulement "historiquement" ${ }^{28}$. Par contraste, la vie auto-affectante que thématise Henry ne connaît pas d'autre histoire que celle de la modulation de ses propres affects : une processualité, donc, rigoureusement immanente, dans laquelle s'épuise toute dynamique de l'expérience de soi. Pour Henry, l'histoire originelle est "passage des tonalités les unes dans les autres", « devenir immanent des tonalités subjectives de l'existence ${ }^{29}$, ce devenir qu'il appelle aussi, d'une formule saisissante, «l'historial de $l^{\prime}$ absolu $»^{30}$. Cette description, qui fait peut-être penser davantage à une vie éternelle qu'à une vie temporelle à proprement parler, est incontestablement tributaire de certains présupposés très lourds : à la suite de Husserl et de Heidegger, Henry continue à attribuer au temps une structure extatique, mais dans cette structure il reconnaît finalement une extériorisation, voire une aliénation de la vie. Alors que, dans sa critique du traitement husserlien de l'impression originaire, Henry s'insurgeait contre la déréalisation de cette dernière par son inscription dans le réseau intentionnel des rétentions, il semble paradoxalement aboutir lui-même à une déréalisation du temps et retrouver donc, dans une certaine mesure, la position aporétique de Brentano, pour qui le temps finissait par se réduire à un ens imaginarium - position dont la critique, comme on le sait, a été le point de départ de Husserl dans ses Leçons sur la conscience intime du temps.

En réduisant l'histoire à la modulation immanente des affects, Henry se prive sans doute des moyens de rendre compte d'une autre historicité, mondaine non pas nécessairement au sens de "l'histoire universelle », mais au sens plus événementiel de ce que Heidegger appelle la Vollzugsgeschichte, l'histoire de l'accomplissement, et qui lui apparait comme indispensable pour aborder la situation et l'expérience des premiers chrétiens. Il est en effet remarquable que Michel Henry et le jeune Heidegger aient donné, au sein de leurs phénoménologies de la vie, une place si importante aux expériences relatées par les écrits néo-testamentaires. En suivant sur ce point Dilthey, Heidegger voit dans le proto-christianisme le «paradigme historique le plus profond $»^{31}$ pour approcher la vie facticielle. La mobilité intrinsèque de la vie, son inquiétude et sa tension vers le point de paroxysme de son devenir - la parousie - s'y manifestent au plus

\footnotetext{
${ }^{28}$ GA 9, p. 38.

${ }^{29}$ EM, p. 842.

${ }^{30} E M$, p. 837. Cf. GP, p. 370 : « histoire de l'affectivité en tant que son historial ».

${ }^{31}$ GA 58, p. 61.
} 
haut degré, tout comme son indissoluble appartenance à son propre temps ou le fait qu'elle vit toujours son temps. En préférant, selon toute apparence, la contemplation johannique à la narration paulinienne ${ }^{32}$, Henry est naturellement enclin à minimiser le rôle déterminant de la situation - et a fortiori de la situation historique - pour l'expérience de la vie des premiers chrétiens, qui renvoie à une sphère $\mathrm{d}^{\prime}$ accomplissement de la vie sans doute plus large que celle de l'autoaffection $^{33}$. N'est-ce pas, en effet, en ce point précis que se révèle le caractère mondain de l'expérience de la vie?

\section{Le monde de la vie}

S'il semble difficile de séparer histoire et monde, cela ne veut pas dire qu'une conclusion d'allure hégélienne qui réduirait toute historicité à la Weltgeschichte s'impose ici inexorablement. Lorsque l'on parle de l'expérience de la vie, ce qui est en question, c'est plutôt, nous l'avons déjà suggéré, l'historicité du soi vivant. S'il y a une dynamique, une processualité de l'expérience de soi de la vie, un monde devrait lui correspondre ne serait-ce que dans l'acception minimale de sa sphère d'accomplissement. Or, du point de vue d'une histoire qui ne se comprend que comme modulation immanente des affects, il devient redoutablement difficile de rendre compte de quelque chose comme un "monde de la vie » sous une forme autre que celle d'un supposé "monde intérieur » (expression qu'à notre connaissance Henry n'emploie pas). Quand il y a dualité de l'apparaître, quand l'apparaître sensible du monde est radicalement hétérogène par rapport à celui, affectif, de la vie, comment penser le rapport entre vie et monde?

Tout en soulignant à maintes reprises «l'indigence ontologique de l'apparaître du monde $»^{34}$ comme «milieu de l'irréalité ${ }^{35}$, Henry ne

\footnotetext{
32 Cet effet de contraste est pourtant à relativiser aussitôt. Voir en ce sens : Martina Roesner, "Logos und Anfang. Zur Johanneischen Dimension in Heideggers Denken ", in Heidegger und die christliche Tradition. Annäherungen an ein schwieriges Thema, éd. N. Fischer et F.-W. von Herrmann, Hambourg, Meiner, 2007, p. 33-54. Pour une critique de l'approche anhistorique du christianisme par Michel Henry, voir l'étude d'Yves-Marie Blanchard, " Michel Henry, lecteur de saint Jean », in Phénoménologie et christianisme chez Michel Henry, éd. Ph. Capelle, Paris, Cerf, 2004, p. 83-94.

${ }^{33}$ Pour Henry, accomplissement de la vie et auto-affection sont rigoureusement synonymes. Voir PV I, p. 127 : "Que veut dire "la vie s'accomplit" ? Qu' elle s'auto-affecte [...]. Voilà pourquoi aucun accès au vivre de la vie n'est possible dans l'apparaître d'un monde ».

${ }^{34}$ Voir, entre autres, le $\S 4$ d'Incarnation : «La crise de la phénoménalité chez Heidegger. L'indigence ontologique de l'apparaître du monde ».

${ }^{35} E M$, p. 564.
} 
peut pas ignorer la nécessité d'une fondation et d'une explicitation du monde de la vie. S'il s'agit là d'une tâche léguée aussi par le dernier Husserl, il est à noter que Michel Henry ne fait pas sien le sens husserlien de la Lebenswelt ${ }^{36}$ - le monde perçu, le monde de l'expérience sensible et de l'extériorité visible, qui est au contraire à soumettre à une " critique radicale ${ }^{37}$. Un monde de la vie qui soit véritablement tel et qui se trouve en consonance avec son propre concept phénoménologique de vie devrait bien plutôt nommer le monde de l'auto-affection, un «monde affectif $»^{38}$, voire "charnel» (mais là encore, non pas au sens de la « chair du monde » merleau-pontienne qui revient au bout du compte à une « absolutisation du Sensible $»^{39}$ ). $S^{\prime}$ il est vrai que Michel Henry n'hésite pas parfois à affirmer sans réserves le caractère acosmique ${ }^{40}$ de la vie et de son auto-affection, il reconnaît en même temps que l'hétérogénéité phénoménologique de la vie et du monde est à surmonter par une fondation plus radicale de la Lebenswelt: "Parce que, en ma chair, je suis la vie de mon corps organique, je suis aussi celle du monde. C'est en ce sens originel, radical, que le monde est monde-de-la-vie, une Lebenswelt ${ }^{41}$.

On voit ici que Michel Henry fait du soi vivant le centre et, pour ainsi dire, le cœur du monde ${ }^{42}$. Le monde est monde de la vie seulement dans la mesure où le soi vivant vit en lui. C'est à cette même thèse que revient, au bout du compte, la position du jeune Heidegger, telle qu'elle s'exprime dans un des concepts les plus importants de son herméneutique de la facticité : celui de Selbstwelt. Le monde du soi (nous préfèrons cette traduction à celle par monde

\footnotetext{
${ }^{36}$ Cf. $C M V$, p. 54 : «Ce monde d'avant la science, le monde de la vie (Lebenswelt), la vie pourtant ne se montre jamais en lui ». Voir aussi Michel Henry, Phénoménologie de la vie, tome IV : Sur l'éthique et la religion, Paris, PUF, coll. "Épiméthée », 2004, p. 110 : « Le monde est monde-de-lavie (Lebenswelt) mais dans un tout autre sens que le sens husserlien. Chez Husserl, le monde-dela-vie signifie que le monde est donné à la sensibilité à laquelle se réfère en dernier lieu tout objet possible, les idéalités scientifiques par exemple. De toute façon, le monde reste une plage de la transcendance. Dans une phénoménologie de la vie, au contraire, le monde se dédouble ». ${ }_{37}$ Cf. I, § 17 (« La critique radicale du monde sensible»), p. 139: «Monde-de-la-vie (Lebenswelt) - selon l'expression de Husserl à laquelle nous reconnaîtrons une signification plus radicale que celle qu'il lui donne ». Voir aussi PV III, p. 347.

${ }^{38} E M$, p. 607. L'expression y figure entre guillemets.

${ }^{39}$ Voir sur ce point les analyses du $\S 21$ d'Incarnation, intitulé : "La tentative de surmonter l'opposition du corps sentant et du corps senti : la problématique du dernier Merleau-Ponty et l'absolutisation du sensible" .

${ }^{40}$ Par exemple, Michel Henry, C'est moi la vérité, Paris, Seuil, 1996 - abrégé par CMV-, p. 134, et «La Vie», in Michel Henry, op. cit., p. 94. Voir aussi le $\S 34$ de L'essence de la manifestation, "Conscience du monde et conscience sans monde».

${ }^{41} I$, p. 216.

42 Pour transposer la belle expression de Merleau-Ponty : «Le corps propre est dans le monde comme le cœur dans l'organisme » (Phénoménologie de la perception, Paris, Gallimard, 1945, p. 272).
} 
propre) n'est certainement pas un monde qui ne ménage aucune place à l'ipséité, comme Henry le reprochait au concept de monde mis en place par l'analytique existentiale du Dasein, mais nomme au contraire la concentration du monde de la vie autour du soi. Avec le monde ambiant (Umwelt) et le monde commun (Mitwelt), il correspond à une certaine perspective sur le monde de la vie. C'est assurément la Selbstwelt qui exprime le mieux, au sujet de la vie facticielle, l'entrelacement constitutif entre le soi et le monde. Mais tout comme Henry, le jeune Heidegger fait ultimement du soi la source du sens et de l'effectivité du monde. Lorsqu'on lit par exemple : «Le soi dans l'accomplissement actuel de l'expérience de la vie, le soi dans l'expérience de soi-même est l'effectivité originaire ${ }^{43}$, on comprend mieux pourquoi (le jeune) Heidegger est en un sens légitimement susceptible de subir le reproche d'acosmisme (ou du moins celui d'idéalisme). Peut-être ce qui permet, au niveau de son herméneutique de la vie facticielle, de prendre sa défense contre ce chef d'accusation est finalement le fait qu'il ne réduit pas le monde de la vie au monde du soi, qu'il essaie de tenir ensemble et d'articuler monde ambiant, monde commun et monde du soi en tant que "genuine Lebenswelten » ou "Sorgenswelten ${ }^{44}$, mondes du souci.

La place insigne du concept de Lebenswelt chez le jeune Heidegger est en tout cas la preuve incontestable de son refus d'opérer une quelconque scission entre la vie et le monde. Dans cette perspective, l'expérience de soi de la vie est immédiatement expérience du monde, car " toute vie vit dans un monde » et «notre vie n'est comme vie que dans la mesure où elle vit dans un monde $»^{45}$. Cette inséparabilité est même affirmée parfois sous la forme d'une quasi-identité : " notre vie est notre monde », ou encore "notre vie est le monde dans lequel nous vivons, en direction duquel et à l'intérieur duquel se déploient les tendances de la vie ${ }^{46}$. Le monde apparaît ainsi, de façon significative, comme l'espace de jeu projeté par les tendances inhérentes au soi vivant, de même que l'histoire surgit, dans sa forme originelle, à partir de la mobilité intrinsèque de ce dernier. Les deux aspects ne sont d'ailleurs pas étrangers l'un à l'autre, comme l'illustre éminemment le concept de situation : la situation est toujours mondaine et historique ou temporelle du même coup.

\footnotetext{
${ }^{43}$ GA 59, p. 173.

${ }^{44}$ GA 58, p. 98 et GA 61, p. 94.

${ }^{45}$ GA 58, p. 36 et 34 .

${ }^{46}$ GA 58, p. 33 et 34 .
} 
La vie facticielle, précisément en tant que facticielle, est donc toujours une vie située, et sa situation n'est pas à prendre au sens d'une limitation ou d'une contrainte, ce pourquoi Heidegger peut parler sans contradiction d'une « auto-suffisance (Selbstgenügsamkeit) » de la vie facticielle. De façon suggestive, lorsque Michel Henry invoque à son tour, comme il le fait à maintes reprises, l'auto-suffisance de la vie, c'est en visant une démarcation entre l'expérience pure de l'auto-affection et l'apparaître mondain ${ }^{47}$. Pour le jeune Heidegger en revanche, l'auto-suffisance de la vie exprime précisément sa capacité $\mathrm{d}^{\prime}$ avoir un monde ${ }^{48}$, voire, si l'on peut oser ici cette expression, de faire monde (nous ne sommes peut-être pas très loin ici de la Weltbildung de la fin des années 20). L'auto-suffisance de la vie peut ainsi apparaître comme la conséquence de sa mondanéisation, et non pas comme l'expression de son caractère acosmique.

Si la vie facticielle n'est assurément pas dépourvue de monde ou weltlos, elle pourrait néanmoins être encore en un certain sens acosmique, car son monde n'est pas à proprement parler un monde d'objets ou de réalités, n'est pas la nature ou le $\operatorname{cosmos}^{49}$ : ce au milieu de quoi la vie vit en ayant un monde, en vivant dans un monde, ce sont bien plutôt, pour Heidegger, des unités de sens qu'il appelle des significativités. C'est en ce point que se dévoilent les raisons profondes qui font que sa phénoménologie de la vie facticielle prend la forme d'une herméneutique : dans sa facticité, la vie est traversée par le sens, elle vit dans l'élément du sens. Nous avons déjà mentionné le fait qu'un des caractères décisifs à l'aide desquels Heidegger définit la facticité est l'expressivité50. La mise en avant de la dimension du sens n'est certes pas une concession faite à une approche théorique ou objectivante de la vie, car le sens en question n'est ni une idéalité, ni le corrélat d'une Sinngebung. Si, pour éviter l'écueil d'une contamination de la vie affective par l'ordre des objectivités idéales, Henry s'autorise parfois à affirmer, de façon provocante, que « la vie n'a pas de sens", il suppose ce faisant que le sens se réduit à une entité idéale, au « corrélat noématique d'une intentionnalité » ${ }^{51}$, sans considérer l'hypothèse d'un sens pré-théorétique intrinsèque à la vie

\footnotetext{
${ }^{47}$ Cf. CMV, p. 134 : «Dans le concept d'auto-affection comme essence de la vie est impliqué son acosmisme, le fait que, n'étant affectée par rien d'autre ou d'extérieur, elle s'accomplit en soimême dans la suffisance absolue de son intériorité radicale - n'éprouvant que soi, n'étant affectée que par soi, avant tout monde possible et indépendamment de lui ».

${ }^{48}$ Cf. GA 58, p. 207.

${ }^{49}$ Cf. GA 61, p. 86.

${ }^{50}$ Cf. GA 58, p. 257.

51 GP, p. 358 et 359.
} 
(comme celui vers lequel fait signe, d'ailleurs, l'idée même d'un «langage de la vie ${ }^{52}$ ). C'est précisément ce que le jeune Heidegger s'efforce de penser à travers le concept de significativité (Bedeutsamkeit). Ce concept revient à creuser l'intuition diltheyenne selon laquelle la vie s'exprime immédiatement et peut ainsi être interprétée à partir d'elle-même. Une telle explicitation est le projet et la tâche même de l'herméneutique de la vie. Pour Henry, en revanche, qui assimile le sens au domaine de l'idéalité et le tient ainsi à distance de la sphère d'accomplissement de la vie, de la sphère de l'affectivité, $l^{\prime}$ herméneutique ne peut apparaître que comme une "dérive ${ }^{53}$ et n'est jamais reconnue dans ce qu'elle peut apporter de précieux pour toute thématisation de la vie, à savoir : la présupposition de l'autosuffisance explicative de celle-ci, le fait que la vie peut être sensée sans renvoyer à une Sinngebung54 opérée par la conscience transcendantale ou à des significations extérieures, objectives, préétablies.

La significativité dans laquelle baigne par nature la vie facticielle est aussi l'élément de son expérience du monde. Heidegger offre une description particulièrement suggestive de cette thèse qui revient aussi à une critique implicite de la conception husserlienne de l'expérience :

Lorsque je bois du thé je prends la tasse dans la main. Ce n'est pas que je saisis quelque chose de coloré ou même, des données de sensation présentes en moi, comme chose et cette chose comme tasse, déterminée dans l'espace et dans le temps, comme quelque chose qui se donne dans une succession de perceptions et qui pourrait aussi, éventuellement, ne pas exister. "Ma tasse dont je bois": sa réalité effective s'accomplit dans la significativité, elle est elle-même. Je vis facticiellement toujours pris dans la significativité (bedeutsamkeitgefangen) ${ }^{55}$.

\footnotetext{
52 Par le rejet de la catégorie herméneutique du sens, il n'est en effet pas question pour Henry d'affirmer l'ineffabilité de la vie et d'élaborer sa phénoménologie de la vie comme une sigétique, car il reconnaît de façon décisive l'existence d'un langage de la vie. Le dernier livre paru de son vivant, Paroles du Christ (Paris, Seuil, 2002), en est la preuve par excellence, même s'il montre aussi que le langage de la vie reste conçu par opposition avec le «langage du monde »opposition qui est, au bout du compte, une nouvelle et peut-être dernière figure de la « dualité de l'apparaître ».

${ }^{53}$ Michel Henry, Phénoménologie matérielle, Paris, PUF, coll. «Épiméthée », 1993, p. 7. Cf. CMV, p. 282 : «De toute façon, la phénoménologie a cédé la place à l'herméneutique, à des commentaires ou, pour mieux dire, à des hypothèses sans fin ». Sur cette critique de l'herméneutique, voir la contribution de Julia Scheidegger: "Michel Henrys Lebensphänomenologie als Hermeneutikkritik », Studia phaenomenologica, vol. IX, 2009, p. 59-82.

${ }_{54}$ Cf. GA 60, p. 220.

${ }_{55}$ GA 58, p. 104, nous traduisons. Voir aussi GA 63, p. 110, où le ton de Heidegger se fait polémique jusqu'au sarcasme.
} 
L'expérience de la significativité est toujours expérience du monde et par là même attestation de la réalité effective ou de l'existence du monde. Le reproche que Heidegger adresse à Husserl dans cet extrait de son cours de 1919-1920 (cours intitulé de façon suggestive Grundprobleme der Phänomenologie) porte en effet sur la description de l'expérience des choses du monde comme expérience externe toujours seulement présomptive, ce qui revient aussi à affirmer la priorité de l'expérience de soi sur l'expérience du monde et leur décalage insurmontable ${ }^{56}$. En logeant l'expérience du monde dans l'élément de la significativité, Heidegger essaie aussi de rétablir l'équilibre entre la Welterfahrung et la Selbsterfahrung. Dans cette perspective, l'opposition même entre deux modes de donation - immanente ou adéquate et transcendante ou inadéquate - devient caduque, car la significativité est le milieu universel de toute expérience, de soi comme du monde. (Inversement, on peut apercevoir dans la dualité de l'apparaître instituée par Henry une radicalisation, voire une absolutisation des deux volets de la Gegebenheit husserlienne.)

La dimension du sens peut fonctionner, non seulement comme une médiation entre le soi et le monde, mais aussi comme un principe d'intelligibilité au niveau de la vie, comme une démarcation puissante et efficace par rapport à un concept purement biologiste de vie. Elle permet sans doute aussi de rendre compte du déploiement de la vie et de ses tendances, jusqu'à son écriture - visible ou non - dans une biographie qui est plus et autre chose qu'un courant vital aveugle, anarchique et anonyme : c'est la significativité, comme l'écrit le jeune Heidegger, qui fournit la " possibilité de la motivation ${ }^{57}$ et, avec elle, l'enchaînement ordonné des tendances vitales. Plus loin encore, seule cette présence du sens à même la vie prouve la nécessité interne et la légitimité de quelque chose comme une herméneutique de la vie : la tâche de cette dernière n'est pas de plaquer sur son objet des significations extérieures et étrangères, mais d'expliciter et d'amener à l'expression sa significativité latente.

\section{En guise de conclusion : le corps de la vie et son monde}

Le sens est l'élément commun de l'expérience de soi et de l'expérience $\mathrm{du}$ monde. Une même fonction d'unification de ces deux

\footnotetext{
${ }^{56}$ Voir en ce sens Husserl, Erste Philosophie (1923-1924), Husserliana, tome VIII, éd. Rudolf Boehm, La Haye, Martinus Nijhoff, p. 453.

57 GA 58, p. 217.
} 
registres de l'expérience de la vie pourrait sans doute être attribuée à l'être corporel. Sur ce terrain, c'est Michel Henry qui semble offrir les plus riches descriptions, car sa phénoménologie de la vie est aussi une phénoménologie de l'incarnation. En effet, se sentir, c'est immédiatement avoir un corps, mieux : être un corps, et la chair se définit même par le fait d'être une " auto-impressionnalité vivante ${ }^{58}$. Sans se présenter à son tour comme une phénoménologie de l'être incarné, l'herméneutique de la facticité du jeune Heidegger n'est cependant pas étrangère à toute considération du corps. La corporéité est reconnue comme une "couche fondamentale (Grundschicht)» de la vie, dont la considération est d'une «importance fondatrice (von grundlegender Bedeutung) ${ }^{59}$. Cette prise en compte révèle que « la constitution fondamentale de l'expérience de la vie est donnée à travers le rapport nécessaire à la corporéité $»^{60}$. Une tâche demeurée cependant en suspens chez les deux auteurs est néanmoins celle $d^{\prime}$ articuler corps et monde, ou encore d'ancrer dans la chair ellemême l'expérience du monde (voire, la Weltmöglichkeit, pour reprendre l'expression de la fameuse note de travail de mai 1960 accompagnant Le visible et l'invisible $\left.{ }^{61}\right)$. C'est à penser l'être-au-monde comme corporel et l'être-incarné comme être-au-monde, en vue d'une co-appartenance encore plus profonde de l'expérience de soi et de l'expérience du monde, qu'une phénoménologie de la vie digne de son nom doit sans doute encore œuvrer.

\footnotetext{
${ }^{58}$ I, p. 90.

${ }^{59}$ GA 56/57, p. 211 et 210.

${ }^{60}$ GA 56/57, p. 210.

${ }^{61}$ Merleau-Ponty, Le visible et l'invisible, Paris, Gallimard, 1964, p. 304.
} 